\title{
Strong Laws of Large Numbers for Arrays of Rowwise Conditionally Negatively Dependent Random Variables
}

\author{
Ronald Patterson, Tamika Royal-Thomas, Wanda Patterson \\ Department of Mathematics, Winston-Salem State University, Winston-Salem, USA \\ Email: pattersonrf@wssu.edu
}

Received March 13, 2013; revised April 16, 2013; accepted May 18, 2013

Copyright (C) 2013 Ronald Patterson et al. This is an open access article distributed under the Creative Commons Attribution License, which permits unrestricted use, distribution, and reproduction in any medium, provided the original work is properly cited.

\section{ABSTRACT}

Let $\left\{X_{n k}\right\}$ be an array of rowwise conditionally negative dependent random variables. Complete convergence of $n^{-1} \sum_{k=1}^{n} X_{n k}$ to 0 is obtained by using various conditions on the moments and conditional means.

Keywords: Negative Dependence; Complete Convergence

\section{Introduction and Preliminaries}

Concepts of negative dependence have been useful in developing laws of large numbers (cf: Taylor, Patterson and Bozorgnia [1]). Chung-type laws of large numbers for arrays of independent random variables were developed by Taylor, Patterson and Bozorgnia in [2].

Definition 1.1 Two random variables $X$ and $Y$ are pairwise negatively dependent (ND) if

$$
P(X \leq x, Y \leq y) \leq P(X \leq x) P(Y \leq y)
$$

for all $x, y \varepsilon \Re$.

Let $(\Omega, F, P)$ denote a probability space.

Definition 1.2. The sequence of random variables $\left\{X_{n}\right\}$ is said to be conditionally negatively dependent if there exists a sub $\sigma$-field $\zeta$ of $F$ such that for each positive integer $m$

$$
P\left[\bigcap_{i=1}^{m} P\left[X_{i} \varepsilon B_{i} \mid \zeta\right]\right] \leq \prod_{i=1}^{m} P\left[X_{i} \varepsilon B_{i} \mid \zeta\right]
$$

where $P\left[X_{i} \varepsilon B_{i} \mid \zeta\right]$ denotes the conditional probability of the random variable $X$ being in the Boral set $B_{i}$ given the sub- $\sigma$ field $\zeta$. Negatively dependent random variable are conditionally negatively dependent with respect to the trivial $\sigma$-field $\{\phi, F\}$.

Throughout this paper $\left\{X_{n k}: 1 \leq k \leq n, n \geq 1\right\}$ will denote rowwise conditionally independent random variables such that $E X_{n k}=0$ for all $\mathrm{n}$ and $\mathrm{k}$. The major result of this paper shows that

$$
\frac{1}{n} \sum_{k=1}^{n} X_{n k} \rightarrow 0 \text { completely }
$$

where complete convergence is defined (Hsu and Robbins [3]) by

$$
\sum_{n=1}^{\infty} P\left[\left\|\frac{1}{n} \sum_{k=1}^{n} X_{n k}\right\|>\eta\right]<\infty .
$$

Here $\|\cdot\|$ is a function on a separable Banach space to $\Re$. In the next section of this paper, strong laws of large numbers for arrays of rowwise conditionally negatively dependent random variables.

\section{Strong Law for Random Variables}

In this section, several lemmas are used in the proof of the major result. The first lemma will be presented without proof.

Lemma 2.1. Let $X$ and $Y$ be pairwise negatively dependent random variables. Then

$$
\operatorname{cov}(X, Y) \leq 0
$$

Lemma 2.2. Let $X$ and $Y$ be pairwise negatively dependent random variables. Then

$$
\operatorname{var}(X+Y) \leq \operatorname{var}(X)+\operatorname{var}(Y)
$$

Proof: For $X$ and $Y$ negatively dependent, we have by Lemma 2.1

$$
\begin{aligned}
\operatorname{var}(X+Y) & =\operatorname{var}(X)+\operatorname{var}(Y)+2 \operatorname{cov}(X, Y) \\
& \leq \operatorname{var}(X)+\operatorname{var}(Y)
\end{aligned}
$$

Theorem 2.1 Let $\left\{X_{n k}\right\}$ be an array of rowwise conditionally negatively dependent random variables. If 


$$
\text { a) } \sup _{1 \leq k \leq n} E\left|X_{n k}\right|^{2}=O(n)^{\alpha}, \alpha<0
$$

and for all $\eta>0$

$$
\text { b) } \sum_{n=1}^{\infty} P\left[\left|\frac{1}{n} \sum_{k=1}^{n} E_{\zeta} X_{n k}\right|>\eta\right]<\infty
$$

where $E_{\zeta}$ is the conditional expectation with respect to an appropriate $\sigma$-field that gives conditional negative dependence. Then

$$
\frac{1}{n} \sum_{k=1}^{n} X_{n k} \rightarrow 0 \text { completely. }
$$

Proof. Let $\eta>0$ be given. By Markov' inequality

$$
\begin{aligned}
& \sum_{n=1}^{\infty} P\left[\left|\frac{1}{n} \sum_{k=1}^{n} X_{n k}\right|>\eta\right] \\
& \leq \sum_{n=1}^{\infty} P\left[\left|\frac{1}{n} \sum_{k=1}^{n} X_{n k}-E_{\zeta} X_{n k}\right|>\frac{\eta}{2}\right] \\
& +\sum_{n=1}^{\infty} P\left[\left|\frac{1}{n} \sum_{k=1}^{n} E_{\zeta} X_{n k}\right|>\frac{\eta}{2}\right] \\
& \leq C \sum_{n=1}^{\infty} E E_{\zeta}\left|\frac{1}{n} \sum_{k=1}^{n} X_{n k}-E_{\zeta} X_{n k}\right|^{2} \\
& +\sum_{n=1}^{\infty} P\left[\left|\frac{1}{n} \sum_{k=1}^{n} E_{\zeta} X_{n k}\right|>\frac{\eta}{2}\right]
\end{aligned}
$$

By Lemma 2.2, the first term in Equation (7) is bounded by

$$
\begin{aligned}
& C \sum_{n=1}^{\infty} E E_{\zeta}\left|\frac{1}{n} \sum_{k=1}^{n} X_{n k}-E_{\zeta} X_{n k}\right|^{2} \\
& \leq C \sum_{n=1}^{\infty} \frac{1}{n^{2}} E E_{\zeta}\left(\sum_{k=1}^{n}\left(X_{n k}-E_{\zeta} X_{n k}\right)^{2}\right) . \\
& \leq C \sum_{n=1}^{\infty} \frac{1}{n^{2}} \cdot 2^{2} \sum_{k=1}^{n}\left|E X_{n k}\right|^{2} \leq C \sum_{n=1}^{\infty} \frac{n \cdot n^{\alpha}}{n^{2}} \\
& =C \sum_{n=1}^{\infty} n^{-1+\alpha}<\infty
\end{aligned}
$$

The second term of Equation (7) is finite by Equation (6). Thus, the result follows.

\section{Strong Law for Random Elements in $\mathfrak{R}^{\mathrm{m}}$}

Theorem 1.2 can be extended to $\Re^{\mathrm{m}}$. The next definition is a crucial type $p$ inequality used to define a form of negative dependence (cf. Patterson, Taylor, and Bozorgnia [4]).

Definition 3.1. Random elements $X_{k}, k \geq 1$, in a type $p$ Banach space $1 \leq p \leq 2$ are said to be type $p$ negatively dependent if $E\left\|X_{k}\right\|<\infty, 1 \leq k \leq n$ and if there exist a finite positive constant $C$ such that

$$
E\left\|\sum_{k=1}^{n}\left(X_{k}-E X_{k}\right)\right\|^{p}<C \sum_{k=1}^{n} E\left\|X_{k}-E X_{k}\right\|^{p}
$$

for all $n \geq 1$.

Coordinatewise (with respect to the standard basis) negative dependence in $\mathfrak{R}^{\mathrm{m}}$ can yield type 2 negative dependence. To see this for rowwise random elements $X_{n k}$, let $X_{n k}=\left(X_{n k 1}, \cdots, X_{n k m}\right), n, k \geq 1$ be random elements in $\mathfrak{R}^{\mathrm{m}}$ such that $E\left(X_{n k i}^{2}\right)<\infty$ for $1 \leq i \leq m, n, k$ $\geq 1$. Then

$$
\begin{aligned}
& E\left\|\sum_{k=1}^{n}\left(X_{k}-E X_{k}\right)\right\|^{p}=E \sum_{i=1}^{m}\left(\sum_{k=1}^{n}\left(X_{n k i}-E X_{n k i}\right)\right)^{2} \\
& \leq \sum_{i=1}^{m} \sum_{k=1}^{n} E\left(X_{n k i}-E X_{n k i}\right)^{2}=\sum_{i=1}^{n} E \sum_{k=1}^{m}\left(X_{n k i}-E X_{n k i}\right)^{2} \\
& =\sum_{k=1}^{n} E\left\|X_{n k i}-E X_{n k i}\right\|^{2}
\end{aligned}
$$

Theorem 3.1 Let $\left\{X_{n k}\right\}$ be an array of rowwise conditionally coordinatewise negatively random elements in $\mathfrak{R}^{\mathrm{m}}$. If

$$
\text { a) } \sup _{1 \leq k \leq n} E\left\|X_{n k}\right\|^{2}=O(n)^{\alpha}, \alpha<0
$$

and for all $\eta>0$

$$
\text { b) } \sum_{n=1}^{\infty} P\left[\left\|\frac{1}{n} \sum_{k=1}^{n} E_{\zeta} X_{n k}\right\|>\eta\right]<\infty
$$

where $E_{\zeta}$ is the conditional expectation with respect to an appropriate $\sigma$-field that gives conditional negative dependence, then

$$
\frac{1}{n} \sum_{k=1}^{n} X_{n k} \rightarrow 0 \text { completely }
$$

Proof. The proof is similar to that of Theorem 2.1.

\section{REFERENCES}

[1] R. L. Taylor, R. Patterson and A. Bozorgnia, "A Strong Law of Large Numbers for Arrays of Rowwise Negatively Dependent Random Variables," Stochastic Analysis and Applications, Vol. 20, No. 3, 2002, pp. 644-666.

[2] R. Patterson, R. L. Taylor and A. Bozorgnia, "Chung Type Stong Laws for Arrays of Random Elements and Bootstrapping," Stochastic Analysis and Applications, Vol. 15, No. 5, 1997, pp. 651-669.

http://dx.doi.org/10.1080/07362999708809501

[3] P. L. Hu and H. Robbins, "Complete Convergence and the Law of Large Numbers," Proceedings of the National Academy of Sciences of the United States of America, Vol. 33, No. 2, 1947, pp. 25-31. http://dx.doi.org/10.1073/pnas.33.2.25

[4] R. Patterson, R. L. Taylor and A. Bozorgnia, "Strong Laws of Large Numbers for Arrays of Rowwise Conditionally Independent Random Variables," Journal of Applied Mathematics and Stochastic Analysis, Vol. 6, No. 1, 1993, pp. 1-10.

http://dx.doi.org/10.1155/S1048953393000012 